\title{
A new in situ sensor for large-scale snow-cover monitoring
}

\author{
Manfred StÄHli, ${ }^{1}$ Markus STAGHeder, ${ }^{2}$ David GUSTAFsSOn, ${ }^{1}$ Stefan SGHLAEGER, ${ }^{2}$ \\ Martin SCHNEEBELI, ${ }^{1}$ Alexander BRANDELIK ${ }^{2}$ \\ ${ }^{1}$ WSL, Swiss Federal Institute for Snow and Avalanche Research SLF, Flüelastrasse 11, CH-7260 Davos-Dorf, Switzerland \\ E-mail: manfred.staehli@wsl.ch \\ ${ }^{2}$ Forschungszentrum Karlsruhe GmbH, Institute of Meteorology and Climate Research, P.O. Box 3640, D-76021 Karlsruhe, Germany
}

\begin{abstract}
A new in situ sensor for the simultaneous measurement of snow water equivalent, snow density and liquid-water content is presented in this paper. The system consists of radio frequency transmission lines of up to $25 \mathrm{~m}$ length cast in a flat PVC band, which can be set up either horizontally to monitor single snow-layer properties or sloping from a mast to the soil surface to determine vertical snowpack properties. The dielectric coefficient along the flat-band cable is measured with a time-domain reflectometer at high frequencies, and with a low-frequency impedance analyzer. The performance of the sensor system was tested during two winter seasons $(2001-03)$ at the high-alpine test site Weissfluhjoch, Davos, Switzerland. The cable suspension and set-up of the sloping cable was shown to be critical with regard to stability and the formation of unwanted air gaps along the cable. Overall, the sensing system proved quite robust and produced results in agreement with manual snowpack observations.
\end{abstract}

\section{INTRODUCTION}

Avalanche- and flood-warning authorities need precise snow-property data. Hydropower stations in alpine and Nordic countries depend on the snow parameters of their catchments to predict the filling stage of their water-storage reservoirs. The distribution of liquid-water flow paths in a snowpack is also important to know for solute transport and wet-snow avalanche formation.

Radar remote sensing has the potential to provide this information if accurate ground reference measurements are available for calibration. For this purpose, we developed and tested a new in situ sensor system for snow moisture and density determination in a snowpack. The system consists of up to $25 \mathrm{~m}$ long radio-frequency transmission lines cast in a flat

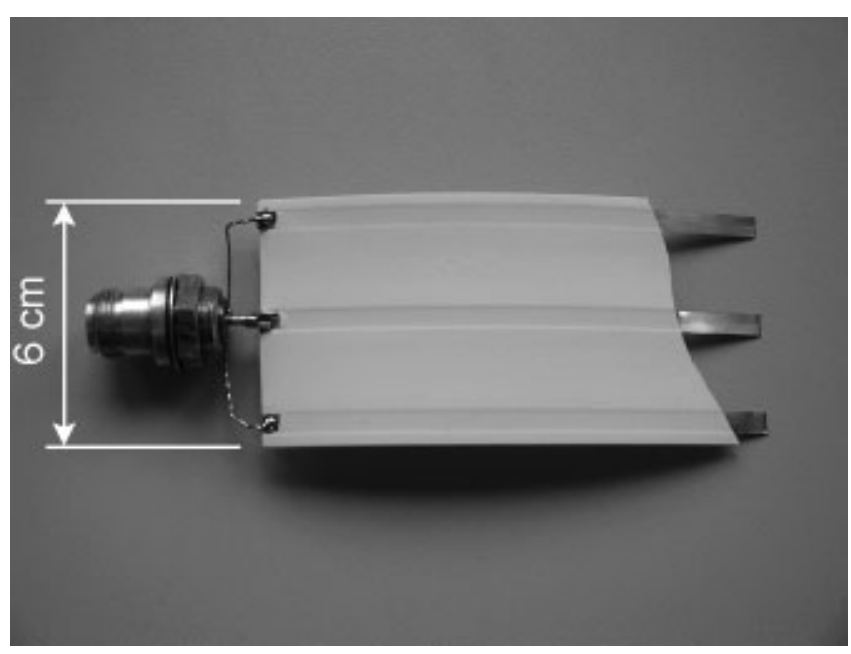

Fig. 1. The insulated flat-band cable (short section with uncovered conductor to show connection and geometry).
PVC band. The dielectric coefficient of the snow along the flat-band cable is measured with a time-domain reflectometer (TDR) at high frequencies and with a low-frequency impedance analyzer. Ice shows significant differences in dielectric properties at high and low frequencies, which enables the determination of the volumetric ice, air and water content, as well as of the snowpack density using measurements at different frequencies. The cable may either be mounted sloping from a mast to the ground to measure vertical snow-profile characteristics or be placed on the snow surface prior to a snowfall to measure the horizontal variations of the dielectric coefficient in the adjacent snow layer.

The objective of this paper is to present the basic principles of the sensor and to show its performance at a highalpine test site in Switzerland during two winter seasons.

\section{MATERIALS AND METHODS}

\section{The snow cable sensor}

The sensor consists of a three-wire flat-band cable with a plastic insulation as shown in Figure 1. The total capacitance $C$ of the equivalent circuit is a non-linear function of the dielectric properties $\varepsilon_{\mathrm{m}}$ of the surrounding material (Huebner and others, 1997):

$$
C=C_{1}+\frac{C_{2} \varepsilon_{\mathrm{m}} C_{3}}{C_{2}+\varepsilon_{\mathrm{m}} C_{3}} .
$$

The parameters $C_{1}, C_{2}$ and $C_{3}$ (Fig. 2) are measured (travel time and wave impedance) and calculated with electromagnetic field simulations. The values for symmetric excitement are compared in Table 1 together with the transmission line inductance.

There is good agreement between the measurement and the calculation. The deviation for $C_{2}$ is due to the 
White polyethylene insulation

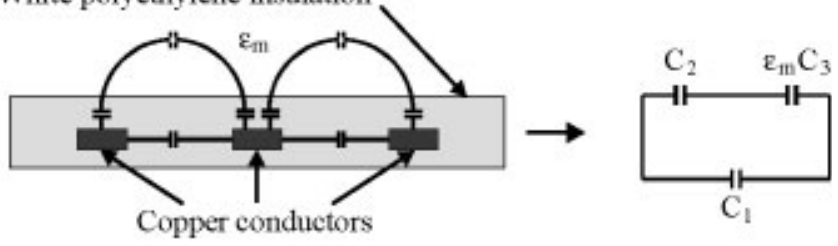

Fig. 2. Flat-band cable sensor cross-section and equivalent circuit.
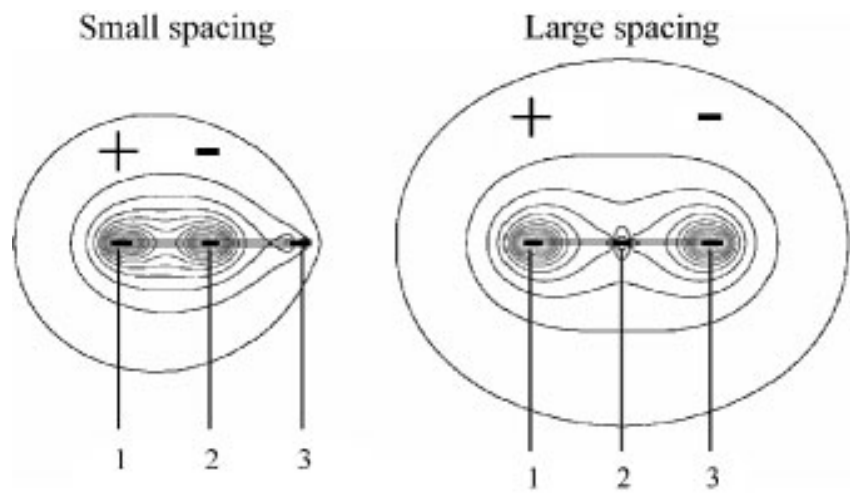

Fig. 3. Electric field extension for small (1+, 2-, 3 open) and large (1+, 2 open, 3-) spacing.

manufacturing process with its tolerance of $\pm 0.1 \mathrm{~mm}$ of insulation thickness.

\section{Air-gap correction}

One of the challenging problems for continuous measurements in snow is the disturbing interaction of the sensor with the surrounding material. An air gap may form around the sensor due to mechanical influences (induced by wind) or partial melting. In order to detect and correct the effect of an air gap, the following procedure is applied. The three-wire sensor cable is measured in two modes, with small and with large spacing as shown in Figure 3.

In the large spacing mode, the air gap has less influence on the measurement of the dielectric coefficient than in the small spacing mode. This influence can be calculated and a correction algorithm can be derived (Brandelik and others, 1998).
Table 1. Comparison of measurement and electromagnetic field calculation

\begin{tabular}{ccc}
\hline Circuit element & Measurement & Field calculation \\
\hline$C_{1}$ & $3.4 \mathrm{pF} \mathrm{m}^{-1}$ & $3.4 \mathrm{pF} \mathrm{m}^{-1}$ \\
$C_{2}$ & $323 \mathrm{pF} \mathrm{m}^{-1}$ & $276 \mathrm{pF} \mathrm{m}^{-1}$ \\
$C_{3}$ & $14.8 \mathrm{pF} \mathrm{m}^{-1}$ & $15.3 \mathrm{pF} \mathrm{m}^{-1}$ \\
$L$ & $756 \mathrm{nH} \mathrm{m}^{-1}$ & $741 \mathrm{nH} \mathrm{m}^{-1}$ \\
\hline
\end{tabular}

In addition to the air-gap correction algorithm, a snow profile reconstruction code has been developed (Becker and others, 2002) which allows the spatial determination of the liquid-water content or the snow-layer density (for situations without liquid water) along the cable sensor.

\section{Field test}

The performance of the sensor system was tested during two winter seasons (2001/02 and 2002/03) at the experimental siteWeissfluhjoch, Davos, Switzerland, located at an altitude of $2550 \mathrm{~m}$ (Fig. 4). The site is snow-covered from late October toJune, and the winter climate is harsh, with minimum air temperatures of down to $-25^{\circ} \mathrm{C}$ and high wind speeds. In both winters, four flat-band cables were set up: one of them sloping from a mast to the soil surface to measure vertical properties of the snow pack (snow depth, density profile, snow water equivalent (SWE)), and three of them horizontally at different depths of the snowpack to capture the spatial variability of liquid-water content and snow density. These horizontal cables were placed on the snow surface at different dates of the winter (Table 2) and followed the natural settling of the snowpack. In addition, in the second winter a dummy sloping cable was mounted at Weissfluhjoch, as well as at a sub-alpine site in central Switzerland (Alptal $(1200 \mathrm{~m})$ ), to examine the influence of the cable on the snow pack during the snowmelt. The electronic measurement devices of the system (TDR cable tester Tektronix 1502B; impedance analyzer HP-VNWA 8712; personal computer $(\mathrm{PC})$ ) were placed in a shelter and connected with $50 \mathrm{~m}$ (first winter) and $35 \mathrm{~m}$ (second winter) coaxial cables to the flat-band sensor.

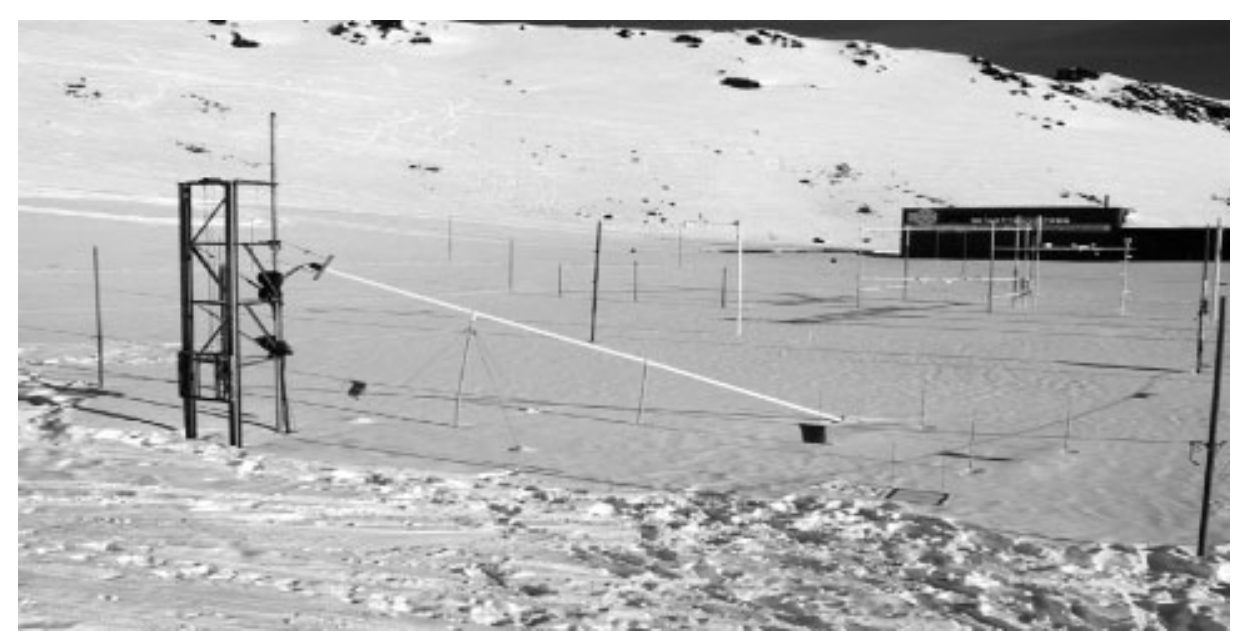

Fig. 4. The Weissfluhjoch test site in January 2002. 
Table 2. Date and snow depth for the days of winter 2001/02 when the horizontal flat-band cables were installed on the snow surface

Horizontalflat-band cable $\quad$ Date of installation $\quad \begin{gathered}\text { Snow depth at day of } \\ \text { installation }\end{gathered}$

$\mathrm{m}$

$\begin{array}{lcc}\text { Cable 1 } & \text { 11 December } & 0.77 \\ \text { Cable 2 } & \text { 28 January } & 1.17\end{array}$

To validate the snow-cable measurements, snow profiles were excavated every 15 days close to the sensor, where the snow density was determined layer-wise. Also, an ultrasonic snow-depth sensor recorded the snow depth continuously, and meltwater outflow from the snowpack was measured in a $2 \times 2$ m lysimeter next to the sloping-cable sensor. Standard meteorological variables were monitored continuously throughout both winter seasons.

\section{RESULTS}

\section{Overall behaviour of the new snow-sensing system}

During the test at Weissfluhjoch we closely inspected the behaviour of the snow-band cable and the electronic system under harsh alpine winter conditions. In particular, the following critical components of the system were examined:
Suspension and set-up of the sloping cable

The original suspension, consisting of a wooden fastener attached with ropes to two iron springs, failed twice during the first winter: after a heavy wind storm in the second half of January, and towards the end of the winter season, when the snow load (maximum SWE: $740 \mathrm{~mm}$ ) became too heavy and a spring broke. Consequently, an improved suspension was designed for the second winter, consisting of a cylinder with a mechanical brake (Fig. 5), which kept a constant pressure on the cable. The suspension adjusted continuously when the snow load increased, by releasing the cable.

The slope angle of the cable sensor is one of the key factors for the formation of an air gap around the flat-band cable, as well as for the bending of the cable inside the snowpack. In the first winter we set up the cable at an angle of $30^{\circ}$ to the ground surface and tried to keep it in its original position by fixing it on three vertical poles. Such poles, however, proved to have negative impacts during the snowmelt by preferentially melting the snow and generating air gaps around the pole. Also a sharp bend of the cable formed at the head of the mid-pole after snowpack settling during the melting period (Fig. 6). In addition, the poles were not stable enough to withstand the heavy snow load and bent considerably towards the end of the season. Consequently, for the second winter we decided to set up the cable at a steeper angle $\left(45^{\circ}\right)$ and to omit the supporting poles. The steeper installation reduced the snow load on the cable, and the cable was kept in its original position during the whole winter. However, we noted increased flickering of the cable in moderate to high wind speeds in the absence of supporting poles. These movements of the cable most likely
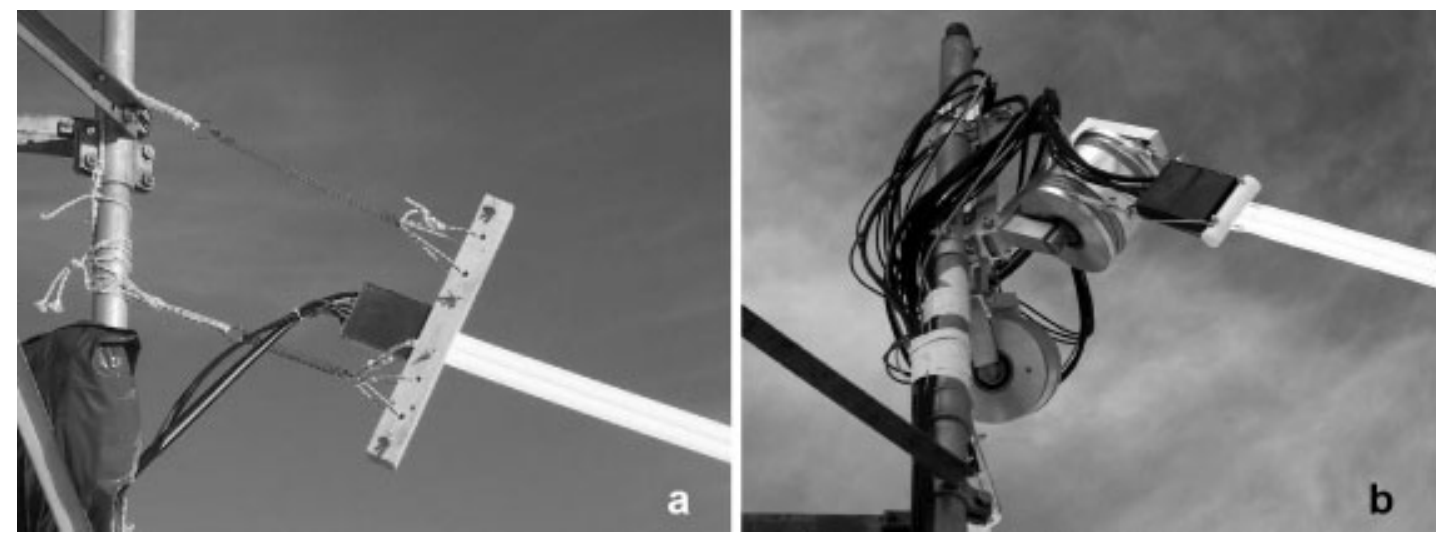

Fig. 5. Suspension of the snow-cable sensor for winter 2001/02 ( a) and winter 2002/03 (b).
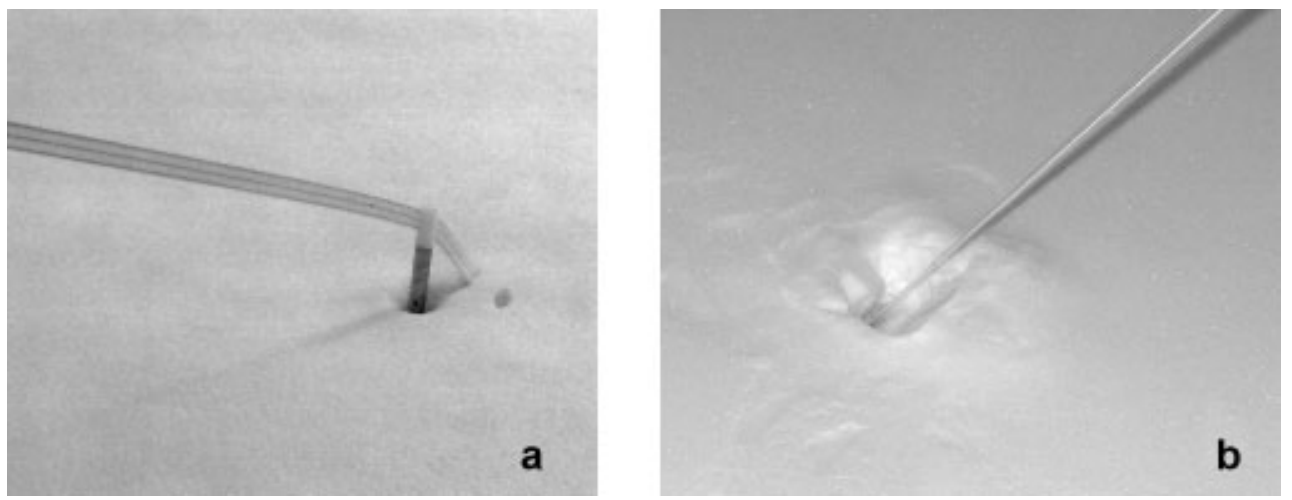

Fig. 6. (a) Preferential melting around the supporting poles and formation of a sharp bend at the head of the pole in winter 2001/ 02. (b) Large air gap at the air-snow interface of the twisted sloping cable in winter 2002/03. 


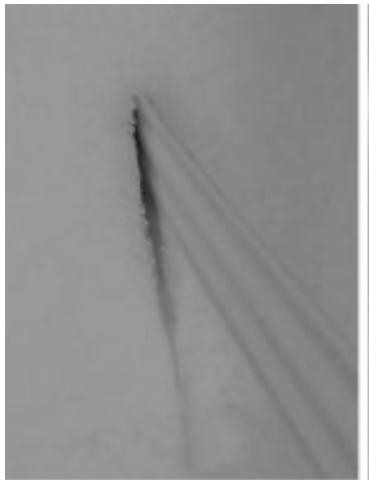

Alptal, 12-Feb, 2003

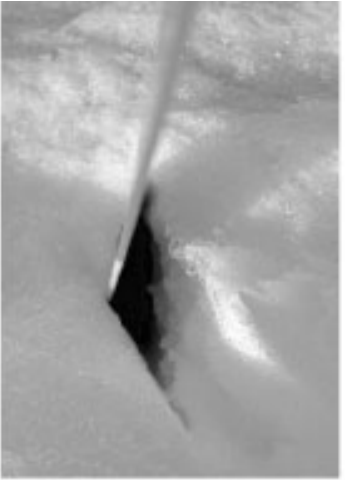

Alptal, 27-Feb, 2003

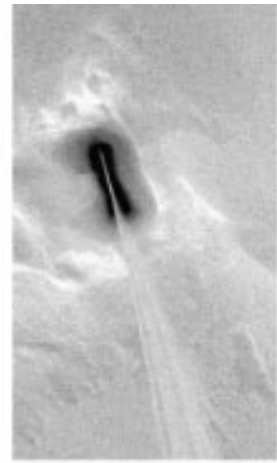

Weissfluhjoch

12-Jan, 2003 sensor 1
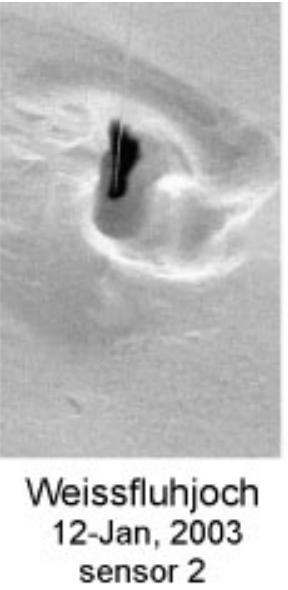

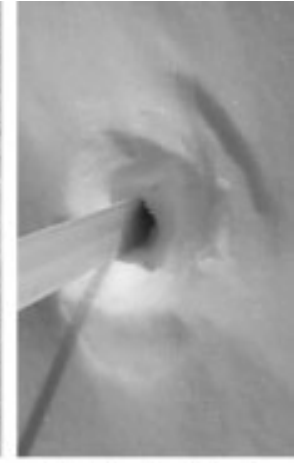

Weissfluhjoch

20-Feb, 2003

Fig. 7. Observations of air gaps at the snow surface in the vicinity of the snow cable sensor.

generated the large air gaps observed at the surface, and may have caused a cable failure during a snowstorm in January 2003. A further complication was a $90^{\circ}$ twist of the cable around its own axes, which may have increased the water flow along the cable (Fig. 6).

Apart from these problems, we believe that the new suspension at a steeper angle has improved the installation, and could be further improved by the combination with springs to reduce the lateral movements of the cable.

\section{Airgap}

Air gaps along the flat-band cable are highly undesirable since they not only falsify the electronic signal, but may also transfer melting water preferentially along the cable. The influence of an air gap on the measurement results can be partially corrected with a correction method (Huebner and others, 1997), but with respect to a potential preferential water transport along the cable it was crucial to thoroughly inspect the size of the air gap and evaluate its importance as a water-flow path.

At both sites the air gap showed up in different sizes and shapes, depending on wind speed, wind direction and melting intensity (Fig. 7). The assumption of a symmetric air gap, which is made in the correction algorithm, was not appropriate for many situations. There were dates (27 February
2003 at Alptal) when an up to $5 \mathrm{~cm}$ opening formed on one side of the band cable, whereas the other side was in close contact with the snow. At the Weissfluhjoch site the air gap was mostly generated by wind. Hence, the compass orientation of the flat-band cable was decisive for the shape of the air gap. We also observed that air gaps were able to fill up again when the wind conditions had changed. Our overall conclusion was that the shape of the air gap at the snow surface was very dynamic and unpredictable. Inside the snowpack, we found no evidence for significant voids around the cable when we excavated the flat-band cable at the sub-alpine site, Alptal, on 1 March 2003. This was at a stage when the snowpack had settled to a density of $290 \mathrm{~kg} \mathrm{~m}^{-3}$ and liquid meltwater had penetrated to the bottom of the snowpack (Fig. 8).

Close inspection of the flat-band cable was also carried out to examine whether the snow band had conducted meltwater preferentially during the snowmelt. A dye tracer had been applied 15 days earlier (prior to the snowmelt) on the snow surface (area indicated in Fig. 8). A vertical profile was excavated along the cable and perpendicularly at the lower end of the cable. No stained water was detected at the lower end of the cable, whereas to the left and right of the cable end considerable stained areas were detected originating from lateral water transport along snow layers. There is evidence that some dye did follow the cable for some distance
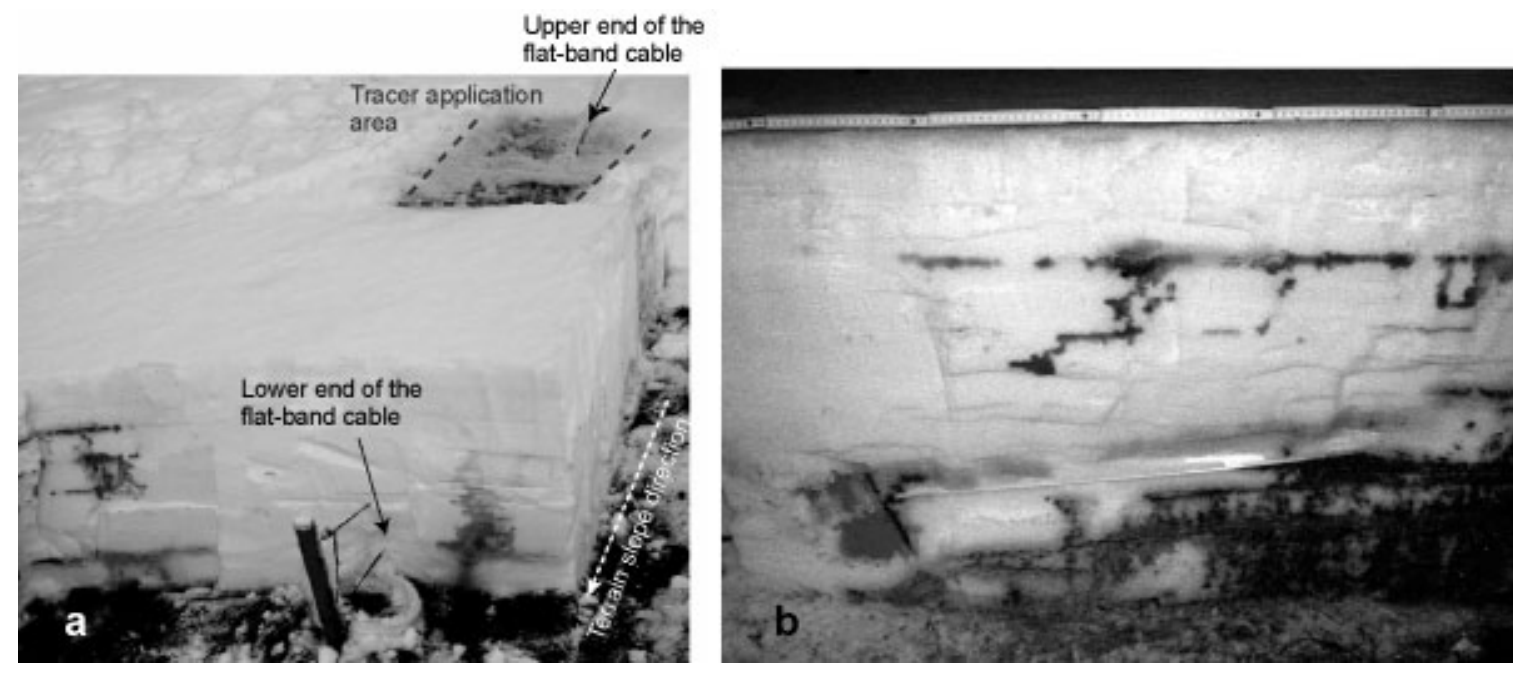

Fig. 8. Dye tracer indicating the water-flow paths during a melt event at the Alptal site. (a) Front view at the cable end, showing no dye tracer near the cable. (b) Vertical cross-section along the flat-band cable. Terrain slope direction is indicated. 


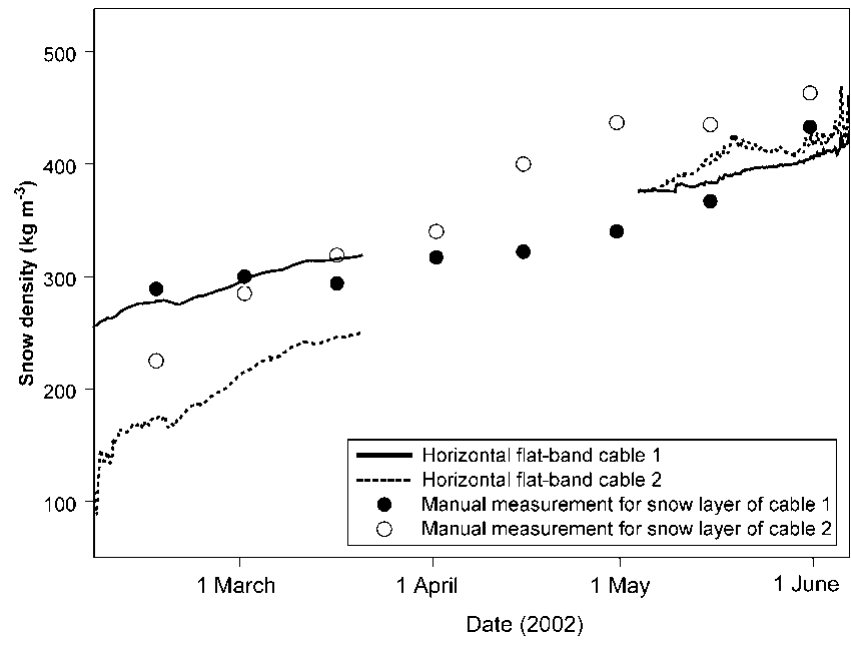

Fig. 9. Measured density along the two horizontal cables during winter 2001/02. Dots show manually determined density of the corresponding snowpack layers. Due to a breakdown of the impedance analyzer, no data were available from late March to early May.

before finding alternative flow paths to the side of and below the cable, but we did not find indications of extensive lateral water flow expressed in terms of intensely stained areas following the direction of the cable. We think that we can conclude in general that water flow along the cable is not greater than the natural lateral water flow in the snowpack.

Concerning thermal effects of the sensor on the surrounding snow, we found some thermal radiation effects of a horizontal cable only once, shortly after it was laid out on the snow surface and when the coverage by fallen snow was very shallow. But as soon as the cable was covered with $>10 \mathrm{~cm}$ of snow, this effect was no longer found.

\section{The electronic system}

Except for some weeks in December 2001, when an erroneous setting prevented the impedance analyzer from running, and in April 2002, when the breakdown of an internal electronic component forced us to replace the impedance analyzer, the electronic system ran more or less failure-free throughout the first winter season. The system uptime was also high in the second winter $(80 \%)$. The automatic start of the measurements occasionally failed at the interface between the controlling PC unit and the Tektronix. Since this occurred mainly at very low temperatures, we think this is a temperature problem of the electronic components of the Tektronix device, which was not built to operate under such low temperatures.

\section{Comparison of snow sensor measurements with manual measurements}

Dielectric constant, bulk snow density and liquid-water content of snowpack (mean value along the cable) were calculated from the raw signals. And the moisture distribution along one of the horizontal cables was reconstructed for different stages of the winter. The natural settling of the snow cover was well reflected in the horizontal cable measurements (Fig. 9). The snow density increased from initially $100-200 \mathrm{~kg} \mathrm{~m}^{-3}$ to approximately $500 \mathrm{~kg} \mathrm{~m}^{-3}$ at the end of winter, which was in accordance with manual snow-density measurements of the corresponding snow layers. The two cables located at two different depths of the snowpack also

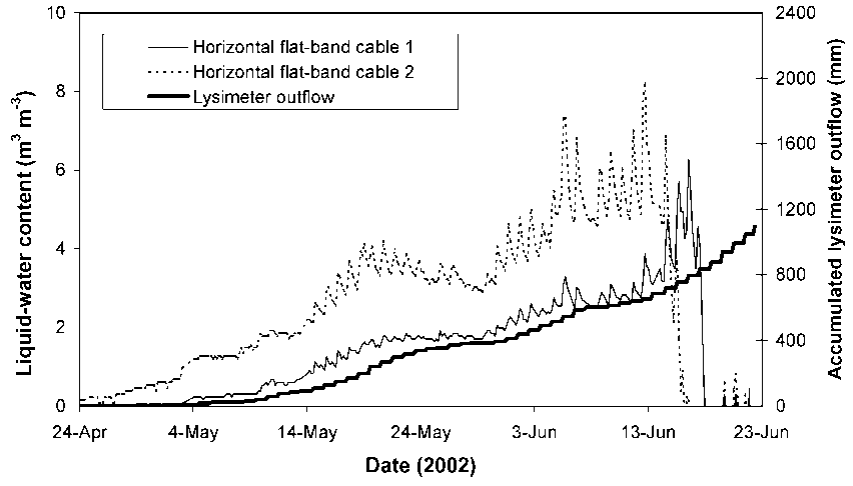

Fig. 10. Liquid-water content (average value along two horizontal cables) during the melting period of spring 2002. Comparison with accumulated meltwater outflow from the lysimeter adjacent to the sensor.

reproduced correctly a higher density in the lower part of the profile in February (cable 1) and a faster compaction in the upper part during the snowmelt (cable 2). However, cable 2 seems to have systematically underestimated density, which we think may be explained by spatial differences in the measurement spots of cable and manual density measurements.

No liquid water was detected with the horizontal cables until the end of April; after that the snowpack had reached its maximum depth (Fig. 10). This is supported by the automated snow temperature measurements, which indicate dry snow conditions and temperatures well below zero before this period. Once the snowmelt set in, we measured the steadily increasing liquid-water content, with the horizontal cables indicating the downward penetration of the wetting front. The upper cable (No. 2) not only reflected an earlier and faster increase in liquid-water content than the lower cable, it also reacted more clearly to the diurnal variation caused by melting during the day and refreezing during the night.

With regard to the spatial variation of liquid water, the horizontal flat-band cable nicely demonstrated the formation of preferential water-flow paths adjacent to the cable (Fig. 11), which is the natural water-transport process in a melting snowpack (Marsh and Woo, 1984). The horizontal cable at about $1 \mathrm{~m}$ below the snow surface indicated in mid-

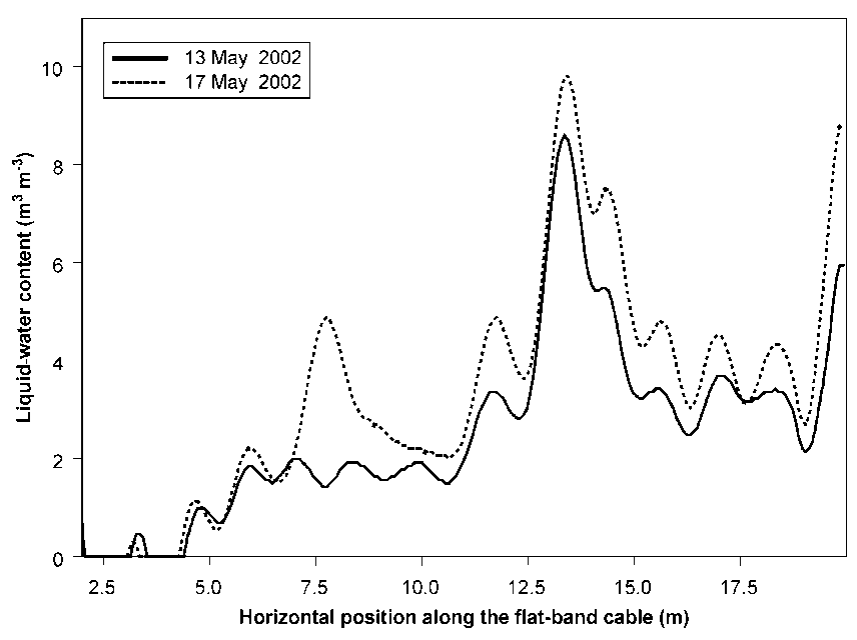

Fig. 11. Spatial variability of liquid-water content along horizontal flat-band cable 2 on 13 and 17 May 2002. 
May an emerging water-conducting zone at $14 \mathrm{~m}$ from the cable end, as well as a newly developing flow finger at $8 \mathrm{~m}$ from the cable end.

\section{DISGUSSION AND GONGLUSIONS}

The measurement results yielded good correspondence of the snowpack density with manual reference measurements taken twice a month at the measurement site. Also, the determination of liquid-water content from measurements of the dielectric constants according to Tiuri and others (1984) gave plausible results, both compared to lysimeter data taken on the test field and with regard to the spatial variation of flow fingers that we normally experience in a natural snowpack. The device's suitability for these measurements has been demonstrated, but the instrumentation set-up needs to be improved to withstand the harsh conditions of alpine winter seasons. This will be a task for future improvements.

We learned that the suspension of the cable sensors is a crucial point in the measurement. For successful measurements it is indispensable to reduce the following sources of error:

Air gaps between cable sensor and surrounding snow must be minimized, although it is possible to correct for minor effects.
The suspension of the cable sensors must allow the cable to settle with the snowpack, but at the same time must be robust enough to withstand heavy winds and snow loads.

\section{ACKNOWLEDGEMENT}

The presented work was carried out within the European Union project SNOWPOWER (fifth framework, NNE5/ 2000/251).

\section{REFERENGES}

Becker, R., A. Brandelik, C. Hübner, W. Schädel, A. Scheuermann and S. Schläger. 2002. Soil and snow moisture measurement system with subsurface transmission lines for remote sensing and environmental applications. In Proceedings of Open Symposium on Propagation and Remote Sensing, Garmisch-Partenkirchen, Germany. URSI Commission-F, Helsinki, 1-14.

Brandelik, A., C. Huebner, G. Doepke and S. Wunderle. 1998. Advanced ground truth for snow and glacier sensing. In IEEE International Geoscience Remote Sensing Symposium, 6-10 July 1998, Seattle, Washington. Proceedings. New York, Institute of Electrical and Electronics Engineers, 1526-1528.

Huebner, C. and 6 others. 1997. A new method for snow moisture sensing. In Proceedings of the EARSeL Workshop on Remote Sensing of Land Ice and Snow, 17-18 April 1997, Freiburg, Germany. Paris, European Association of Remote Sensing Laboratories, 103-109.

Marsh, P. and M.-K. Woo. 1984. Wetting front advance and freezing of meltwater within a snow-cover. 1. Observations in the Canadian Arctic. Water Resour. Res., 20(12), 1853-1864.

Tiuri, M. E., A. H. Sihvola, E. G. Nyfors and M. T. Hallikainen. 1984. The complex dielectric constant of snow at microwave frequencies. IEEE $\mathcal{F}$. Oceanic Eng., OE-9(5), 377-382. 